\title{
RESTENOSIS AFTER PERCUTANEOUS TRANSLUMINAL CORONARY ANGIOPLASTY
}

\author{
-A Histopathological Study Using Autopsied Hearts-
}

\author{
Shin-ichiro Morimoto, M.D., Yasushi Mizuno, M.D., Shinya Hiramitsu, M.D. \\ Kenji Yamada, M.D., Natsuko Kubo, M.D., Masanori Nomura, M.D! \\ Tetsu Yamaguchi, M.D.2, Hidemasa Kitazume, M.D., Ka.zuhisa Kodama, M.D. \\ Hiroyuki Kurogane, M.D., Youichi Shimizu, M.D., Kyolchi Mizuno, M.D? \\ Masao Chino, M.D., Sachiro Watanabe, M.D., Tetsuro Ueda, M.D. ${ }^{10}$ \\ Mitsuyasu Toyoda, M.D., and Morie SeKiguchi, M.D. ${ }^{11}$
}

\begin{abstract}
Restenosis was studied histopathologically by serial step sectioning of 22 coronary arteries from 21 patients in whom percutaneous transluminal coronary angioplasty (PTCA) had been performed ( 9 arteries from patients who had died shortly after PTCA and 13 from those who had died considerably later). Nine of the 13 arteries from the patients who had died long after PTCA were immunohistochemically stained using anti-actin antibody for examination of spindle-shaped cells proliferating in the intima. In the patients who had died shortly after PTCA, all 9 arteries showed fresh thrombus formation. In the patients who had died considerably later after PTCA, however, there was fragmentation of the internal elastic lamina (IEL) in 9 arteries. In each of these 9 arteries, a remarkable proliferation of intimal cells was observed on the intimal side, mainly at the site of the IEL fragmentation. These spindle-shaped cells were identified as smooth muscle cells (SMC) because they stained reddish-brown with Masson's trichrome, and because immunohistochemical staining with anti-actin antibody was also positive. In 2 arteries, proliferation of SMC and elastic fibers was observed on the luminal side of the intima, despite absence of fragmentation in the IEL. Proliferation of SMC in false lumens was identified in 2 patients with medial dissection.

From the above findings, the following 4 forms of restenosis after PTCA have been identified: 1. thrombus formation; 2. proliferation of SMC on the intimal side, mainly around fragmentation in the IEL; 3. proliferation of
\end{abstract}

\author{
Key words: \\ Percutaneous transluminal coronary angioplasty \\ (PTCA) \\ Restenosis \\ Thrombus \\ Internal elastic lamina \\ Smooth muscle cell
}

(Received November 26, 1988; accepted August 9, 1989)

${ }^{1}$ Department of Internal Medicine, Fujita Health University School of Medicine; ${ }^{2}$ Mitsui Memorial Hospital;

${ }^{3}$ Tokyo Metropolitan Bokutoh Hospital; ${ }^{4}$ Osaka Police Hospital; ${ }^{5}$ Hyogo Brain and Heart Center at Himeji;

${ }^{6}$ Sakakibara Heart Institute; ${ }^{7}$ National Defence Medical College; ${ }^{8}$ Ashikaga Red Cross Hospital; ${ }^{9}$ Gifu Prefectural Hospital; ${ }^{10}$ Cardiovascular Center of Sendai; ${ }^{11}$ Tokyo Women's Medical College; Japan

Mailing address: Shin-ichiro Morimoto, M.D., Department of Internal Medicine, Fujita Health University School of Medicine, 1-98 Dengakugakubo, Kutsukake-cho, Toyoake City, Aichi Prefecture 470-11, Japan 
SMC on the luminal side of the intima where there was no fragmentation of the IEL; and 4. proliferation of SMC in dissected false lumen.

The proliferation of SMC on the intimal side of the disrupted IEL was thought to have been a result of migration of SMC from the media to the intima, because SMC proliferation was seen around the disrupted region.

$I^{\prime}$ $\mathrm{N}$ recent years, the development of percutaneous transluminal coronary angioplasty (PTCA) ${ }^{1}$ has been of great benefit to some patients with ischemic heart disease, because improvement without open heart surgery has become possible. The number of patients undergoing PTCA is steadily increasing both in Japan and abroad. Although the procedure is quite advantageous, it presents many problems, one of which is restenosis, which occurs in about $30 \%$ of patients within a few months following
PTCA 2,3 Although the mechanism of luminal enlargement is becoming clear, especially through histopathological studies, ${ }^{4-15}$ the mechanism of restenosis is still unknown. The present study undertook a histological examination of restenosis in PTCA sites in autopsied hearts.

\section{SUBJECTS AND METHODS}

A total of 21 patients, consisting of 9 patients who had died within 7 days after PTCA and

TABLE I CLINICAL OBSERVATIONS IN 21 NECROPSY SUBJECTS WHO DIED AFTER PTCA

\begin{tabular}{|c|c|c|c|c|c|c|}
\hline & $\begin{array}{c}\text { Case } \\
(\text { Age } \cdot \operatorname{Sex})\end{array}$ & $\begin{array}{c}\text { Diag. before } \\
\text { PTCA }\end{array}$ & \multicolumn{2}{|c|}{$\begin{array}{c}\% \text { Diameter } \\
\text { Stenosis }\end{array}$} & $\begin{array}{c}\text { Interval } \\
(\sim \text { Death })\end{array}$ & Cause of Death \\
\hline \multicolumn{7}{|l|}{ Early Death } \\
\hline \multirow[t]{4}{*}{ Emergency } & 1. $(57 \cdot M)$ & $A M I$ & $L M T$ (5) & $100 \rightarrow 75$ & $7 d$ & $C H F, V T$ \\
\hline & 2. $(70 \cdot M)$ & $A M I$ & $L A D(6)$ & $90 \rightarrow 75$ & $1 d$ & $V T$ \\
\hline & 3. $(60 \cdot M)$ & $A M I$ & $L A D(6)$ & $95 \rightarrow 50$ & $10 h$. & Cardiac rupture \\
\hline & 4. $(60 \cdot F)$ & $A M I$ & $L A D(6)$ & $99 \rightarrow 25$ & $6 d$ & Cardiac rupture \\
\hline \multirow[t]{5}{*}{ Elective } & 5. $(61 \cdot M)$ & $A P$ & $L A D(7)$ & $99 \rightarrow 90 \rightarrow 100$ & $1 d$ & AMI, Cardiogenic shock \\
\hline & 6. $(40 \cdot M)$ & $A P$ & $L C X(13)$ & $90 \rightarrow 25 \rightarrow 100$ & $2 d$ & AMI, Cardiogenic shock \\
\hline & 7. $(61 \cdot M)$ & $A P$ & $L A D(7)$ & $75 \rightarrow 20$ & $1 h$. & $A M I, V F$ \\
\hline & 8. $(59 \cdot M)$ & OMI, Ao. Aneu. & $L C X(11)$ & $90 \rightarrow 75$ & $1 d$. & $A M I$ \\
\hline & 9. $(59 \cdot M)$ & AP, Ao. Aneu. & $L C X$ & $80 \rightarrow 50$ & $1 d$ & $A M I$ \\
\hline \multicolumn{7}{|l|}{ Late Death } \\
\hline \multirow[t]{5}{*}{ Emergency } & 10. $(64 \cdot M)$ & $A M I$ & $L A D(6)$ & $100 \rightarrow 70$ & $2 m$ & Pneumonia \\
\hline & 11. $(67 \cdot M)$ & $O M I, A M I$ & $L A D(6)$ & $90 \rightarrow 25$ & $0.5 m$ & $\mathrm{CHF}$ \\
\hline & 12. $(36 \cdot M)$ & $O M I, A M I$ & $R C A(1)$ & $99 \rightarrow 75$ & $2 y .1 \mathrm{~m}$ & $C H F$ \\
\hline & 13. $(54 \cdot M)$ & $A M I$ & $L A D(7)$ & $99 \rightarrow 50 \sim 75$ & $2 y .1 m$ & Gastric cancer \\
\hline & 14. $(65 \cdot M)$ & $A M I$ & $L A D(6)$ & $99 \rightarrow 50$ & $3 m$ & CHF, Pneumonia \\
\hline \multirow[t]{7}{*}{ Elective } & 15. $(56 \cdot M)$ & $A P$ & $L A D(7)$ & $80 \rightarrow 50$ & $7 m$ & Suicide \\
\hline & 16. $(56 \cdot M)$ & $A P$, Lung $C a$. & $L A D(7)$ & $75 \rightarrow 25$ & $2 y .8 m$ & Lung cancer \\
\hline & 17. $(60 \cdot F)$ & AP, Ao. Aneu. & $L A D(7)$ & $90 \rightarrow 10$ & $4 m$ & Pneumonia \\
\hline & 18. $(73 \cdot M)$ & $O M I, A P$ & $\begin{array}{l}L A D(7) \\
L C X(11)\end{array}$ & $\begin{array}{l}99 \rightarrow 25 \\
75 \rightarrow 25\end{array}$ & $4 m$ & MOF, Sepsis \\
\hline & 19. $(71 \cdot M)$ & $O M I$ & $L A D(7)$ & $90 \rightarrow 75$ & $1 y .3 \mathrm{~m}$. & Prostatic cancer \\
\hline & 20. $(57 \cdot M)$ & $A P$ & $L A D(6)$ & $90 \rightarrow 25$ & $1 y$. & Gastric cancer \\
\hline & 21. $(61 \cdot M)$ & $O M I$ & $R C A(2)$ & $80 \rightarrow 50$ & $2 y .11 \mathrm{~m}$ & Gastric cancer \\
\hline
\end{tabular}

Emergency = emergency PTCA; Elective = elective PTCA; $M=$ male; $F=$ female $;$ Diag = diagnosis; $A M I=$ acute myocardial infarction; $O M I=$ old myocardial infarction; AP =angina pectoris; Ca. = cancer; Ao. Aneu. = aortic aneurysm; LMT = left main truncus; LAD =left anterior descending artery; LCX $=$ left circumflex artery; $R C A=$ right coronary artery; $d=$ day; $h=$ hour; $m=$ month; $C H F=$ congestive heart failure; $V T=$ ventricular tachycardia $\quad V F=$ ventricular fibrillation; $M O F=$ multiple organ failure 
12 patients who had died anywhere from 16 days to 2 years and 11 months after the procedure, were the subjects of the present study (Table I). In patient 18, who had died long after PTCA, the procedure had been performed in 2 arteries, namely the left anterior descending artery (LAD) and the left circumflex artery (LCX), making for a total of 22 arteries used in our study.

The clinical diagnoses made before PTCA were myocardial infarction in 13 patients and angina pectoris in 8. PTCA-induced enlargement of stenosed portions was found in coronary arteriograms of the 22 coronary arteries, whereas 2 (patients 5 and 6 ) of them were occluded soon after PTCA. There were only 4 deaths which directly resulted from complications due to PTCA. Some patients died due to causes unrelated to ischemia (e.g., cancer, pneumonia or accidents).

From the 22 arteries from patients who had undergone PTCA, 142 to 1,350 sections were prepared per artery by serial step sectioning. The specimens were stained by Masson's trichrome, Elastica van-Gieson, and hematoxylin-eosin, and were examined under a light microscope. The specimens from 9 of the 13 patients who had died long after PTCA were immunohistochemically stained by the biotin-streptavidin method ${ }^{16,17}$ using anti-actin antibody (Biomedical Technologies Inc.) to examine spindle-shaped cells proliferating on the intima.

In this paper, the following definitions are used: restenosis is a state in which the lumen, once enlarged by PTCA, has narrowed again by any type of mechanism; dissection is a dehiscence along the circumference of a blood vessel (arrow A in Fig. 1), while disruption is a dehiscence running perpendicular through the vascular wall (arrow B in Fig. 1); and intimal desquamation is a state in which a part of the intima is detached completely from the vascular wall (arrow C in Fig. 1).

\section{RESULTS}

Results of the histopathological examinations in representative cases are described herewith.

Case 5: R.O. 61-year-old man

This patient had unstable angina pectoris. Selective coronary arteriography (CAG) showed 90 to $99 \%$ stenosis in the left anterior descending artery (Fig. 2A). When PTCA was performed, a slight enlargement was seen on the right and left

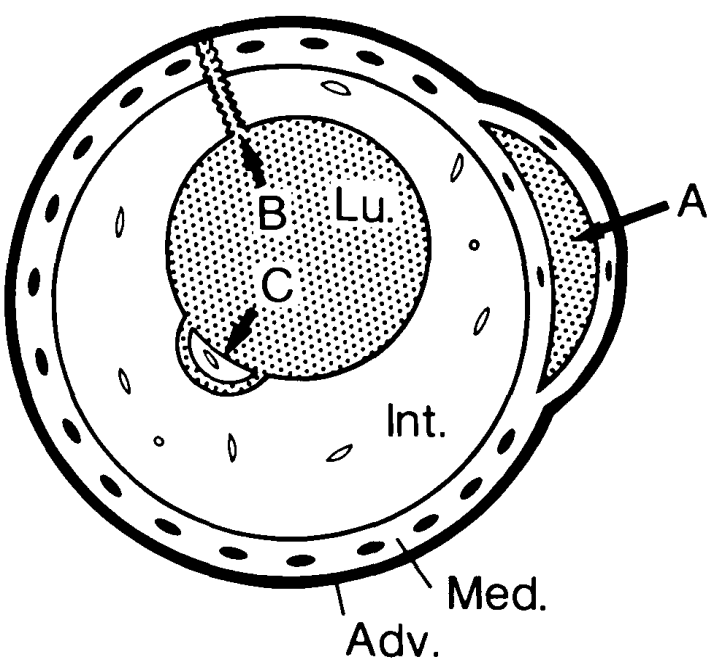

Fig.1. Definitions of terms. A tear along the circumference of the blood vessel (A) is called a dissection. A tear running perpendicular through the vascular wall (B) is called a disruption. $\mathrm{C}$ shows an intimal desquamation.

Lu. = lumen; Int. = intima; Med. = media; Adv. = adventitia

of the arrow, as indicated in Fig. 2B. But when inflations were repeated further, total occlusion occurred, as shown in Fig. 2C. Although aortocoronary bypass was performed immediately, the patient died of acute anterior myocardial infarction 2 days after PTCA.

Autopsy findings: The heart weighed $540 \mathrm{~g}$ and necrosis was found in the left ventricular anterior wall and anterior portion of the ventricular septum (Fig. 2D). In the left anterior descending artery, both intimal and medial disruptions were seen ubiquitously (Fig. 2E), with the result that some parts of the coronary arterial wall consisted only of adventitia (arrow $\mathrm{A}$ in Fig. 2E). Medical dissection was seen widely (arrow D in Fig. 2E), and intimal desquamation was found in some parts (arrow in Fig. 2F). A fresh thrombus was observed in the lumen (Fig. 2E).

\section{Case 10: Y.H. 64-year-old man}

This patient was admitted because of an acute anteroseptal myocardial infarction. CAG performed on the day after onset, revealed an occlusion in the proximal portion of the left anterior descending artery, and PTCA was performed. The stenosis was reduced to about $70 \%$ and the subsequent course was satisfactory, but the patient died 2 months later of aspiration pneumonia. 


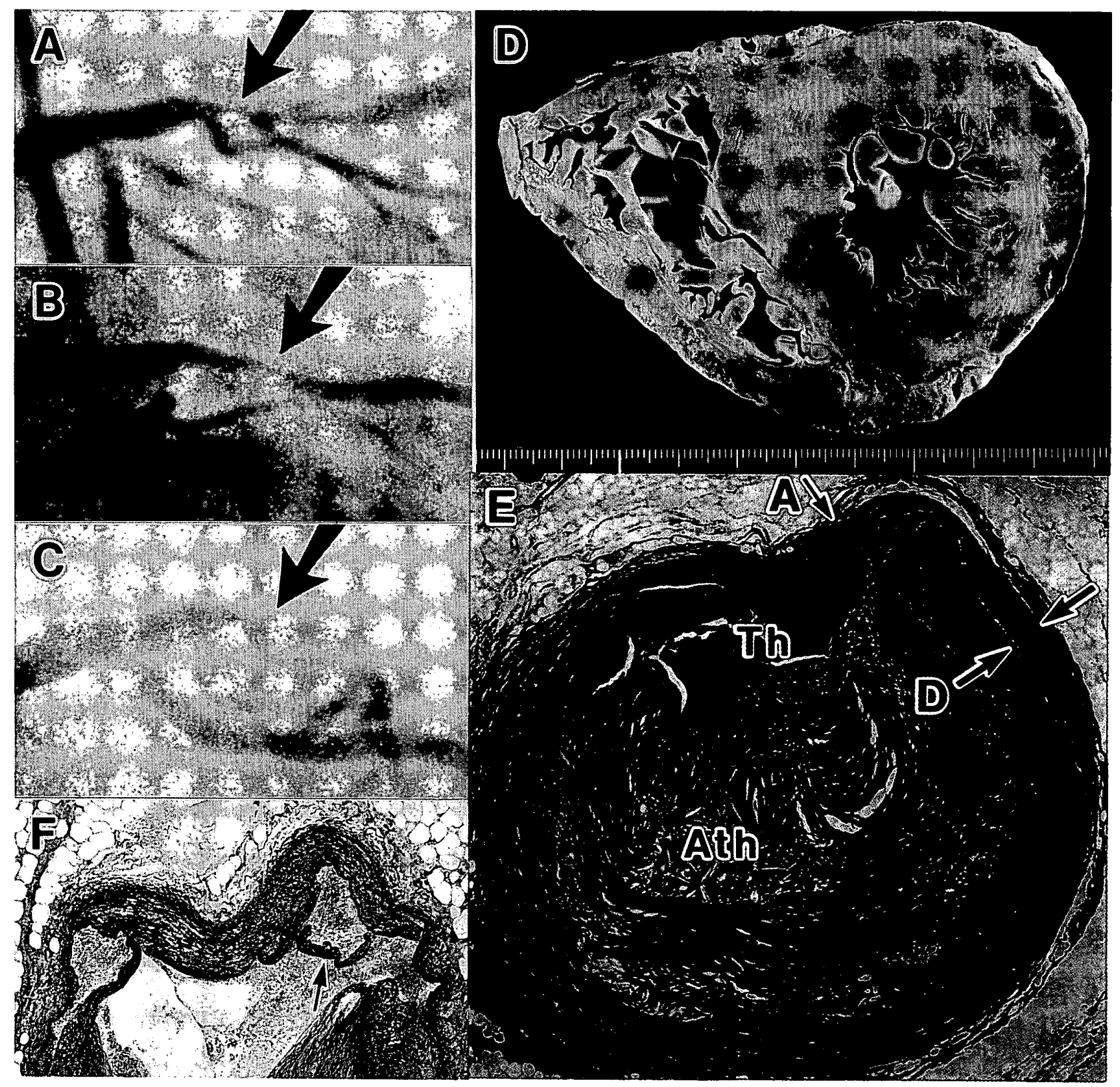

Fig.2. Case 5 A: This patient suffered from angina pectoris. The left anterior descending artery had 90 to 99\% stenosis. B: PTCA slightly enlarged the portion indicated by the arrow. C: Complete occlusion resulted from repeated inflation. D: Infarction is seen in the left ventricular anterior wall and the anterior portion of the ventricular septum. E: Histological picture of the occluded site in the left anterior descending artery (Masson's trichrome stain). A fresh thrombus (Th) is seen. Hemorrhage is observed in the atheroma (Ath). Although the lumen is enlarged by intimal and medial disruption (stars), the coronary arterial wall is partly composed of adventitia (arrow A) only. Medial dissection is also observed (arrow D). F: Intimal desquamation (arrow) is seen. Although this debris might cause a peripheral embolism, it would also slightly enlarge the lumen in this section.

Autopsy findings: The heart weighed $360 \mathrm{~g}$ and both ventricles were slightly dilated. An old infarction was seen in the left ventricular anterior wall and anterior 3 quarters of the ventricular septum. In the proximal part of the left anterior descending artery where PTCA had been performed, intimal and medial disruptions (Fig. 3B, 4A) and medial dissection (starts in Fig. $4 \mathrm{~A}$ and
B) were seen on the arterial wall located opposite the atheroma. The disrupted regions and false lumen were already restenosed by a significant proliferation of intimal cells (Fig. 3C, D, 4D). These spindle-shaped cells could be immunohistochemically stained with anti-actin antibody (Fig. 3E). 


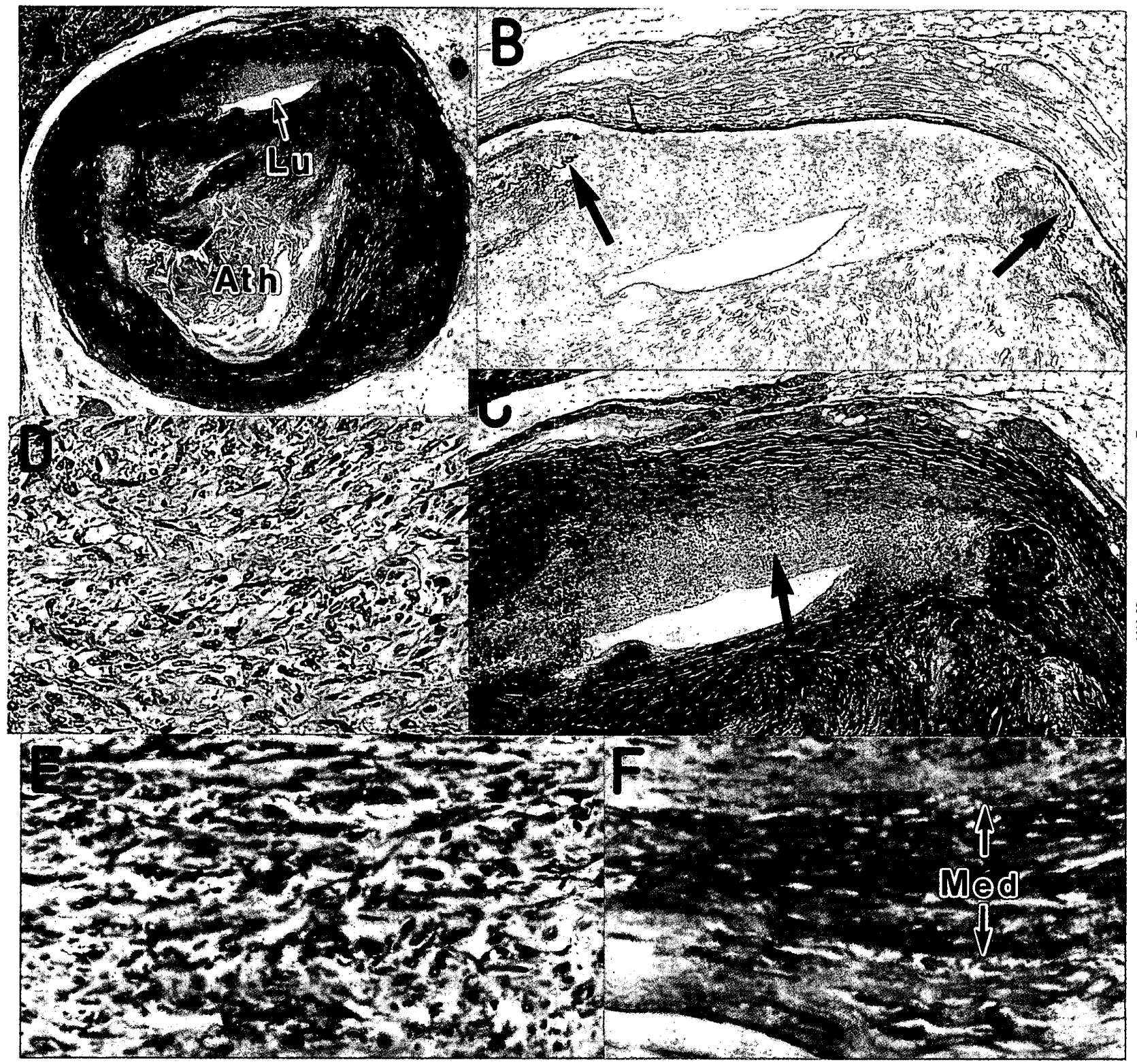

Fig.3. Case 10. This patient was admitted because of acute myocardial infarction. PTCA was performed the day after onset. In the left anterior descending artery, patency was restored, a $100 \%$ narrowing being reduced to $70 \%$. Two months later, however, the patient died of pneumonia. A: Histological picture showing proximal portion of the left anterior descending artery (Lu: lumen, Ath: atheromatous plaque) (Masson's trichrome stain). B, C: Magnified views of the upper portion of A. Intimal and medial disruption is observed (B: Elastica van-Gieson stain). Fragmentation of the internal elastic lamina indicated by arrows in $\mathrm{B}$ is seen. The lumen is already showing restenosis as a result of significant proliferation of intimal cells. D: Magnified view ( $\times 100)$ of the arrow-indicated portion in C. Numerous intimal cells are observed. E: Immunohistochemical stain using anti-actin antibody, for identification of the spindleshaped cells shown in 'D, was positive. F: Coronary artery which did not undergo PTCA. The medial smooth muscle cells are seen to react positively with the anti-actin antibodies (Med: media).

\section{Case 13: Y.O. 54-year-old man}

This patient was hospitalized for acute anterolateral myocardial infarction. CAG was immediately performed, revealing complete occlusion of segment 7 of the left anterior descending artery (Fig. 5A). After the percutaneous transluminal coronary recanalization (PTCR), PTCA was per- formed. Immediately after the procedure the stenosis was reduced to 50 to $75 \%$ (Fig. 5B); within 2 weeks the degree of narrowing was further reduced to 25 to $50 \%$ (Fig. 5C). But 2 months later the patient was readmitted because of acute anterolateral myocardial infarction. The course after his second discharge was satisfactory. 


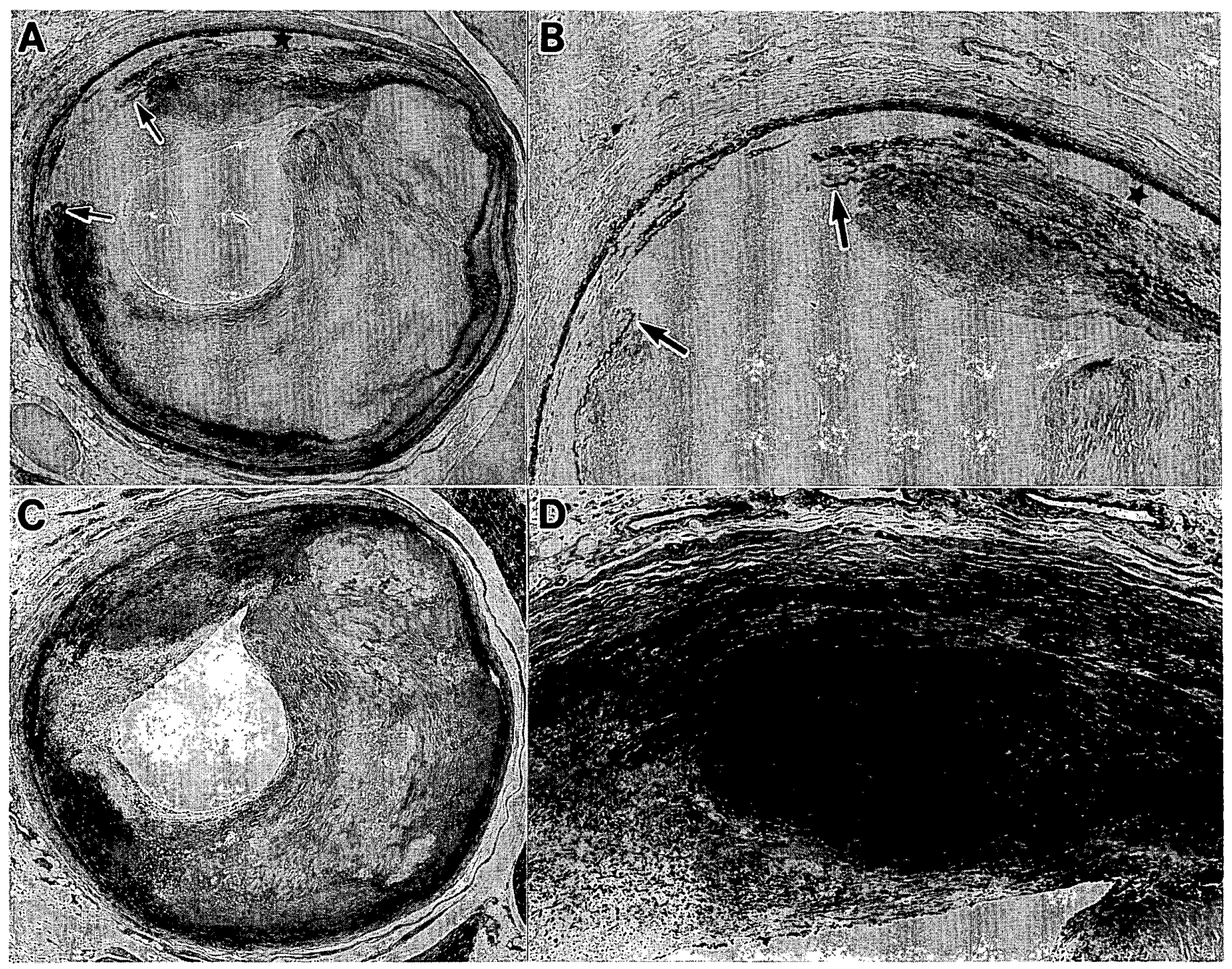

Fig.4. Case 10. Histological picture showing a portion $3 \mathrm{~mm}$ peripheral to Fig. 3. A: Medial disruption (arrows) and dissection (star) are seen (Elastica van-Gieson stain). B: Magnified view of the upper portion of A. C, D: Numerous smooth muscle cells have proliferated at the sites of medial disruption and dissection, causing restenosis (Masson's trichrome stain).

CAG undertaken 1 year after the PTCA performed at the first onset revealed a generally narrow $\mathrm{LAD}$, with 50 to $75 \%$ stenosis of segment 7 (Fig. 5D). The patient died 2 years and 1 month after PTCA, of gastric cancer complicated by carcinomatous pleurisy.

Autopsy findings: In the site in segment 7 of the left anterior descending artery where PTCA had been performed, many small lumina were seen, as in a cross-section of a lotus root (Fig. $5 \mathrm{E})$. In the intima of this site, significant proliferation of smooth muscle cells (arrows in Fig. 5F) and relatively thick elastic fibers (arrows in Fig. 5G) were observed. At a site $8 \mathrm{~mm}$ peripheral to this section, marked proliferation of smooth muscle cells (arrows in Fig. 5H, Fig. 5I) and thick elastic fibers was also seen (arrows in Fig. 5J) on the luminal side of the intima.
Although this cross-section of the coronary artery (Fig. $5 \mathrm{H}$ ) was not necessarily regarded to be the target region of the PTCA, it was considered quite possible that the balloon, having a length of about $20 \mathrm{~mm}$, reached and temporarily enlarged this region. No fragmentation of internal elastic lamina was observed in this patient's PTCA site.

\section{Case 17: M.H. 60-year-old woman}

Angina pectoris and aneurysm of the aortic arch were found in this patient. PTCA performed on segment 7 of the left anterior descending artery (arrow in Fig. 6A) reduced its stenosis from 90\% to about 10\% (arrow in Fig. 6B). The aortic aneurysm was surgically treated on the same day. The postoperative course was satisfactory and the patient was dis- 

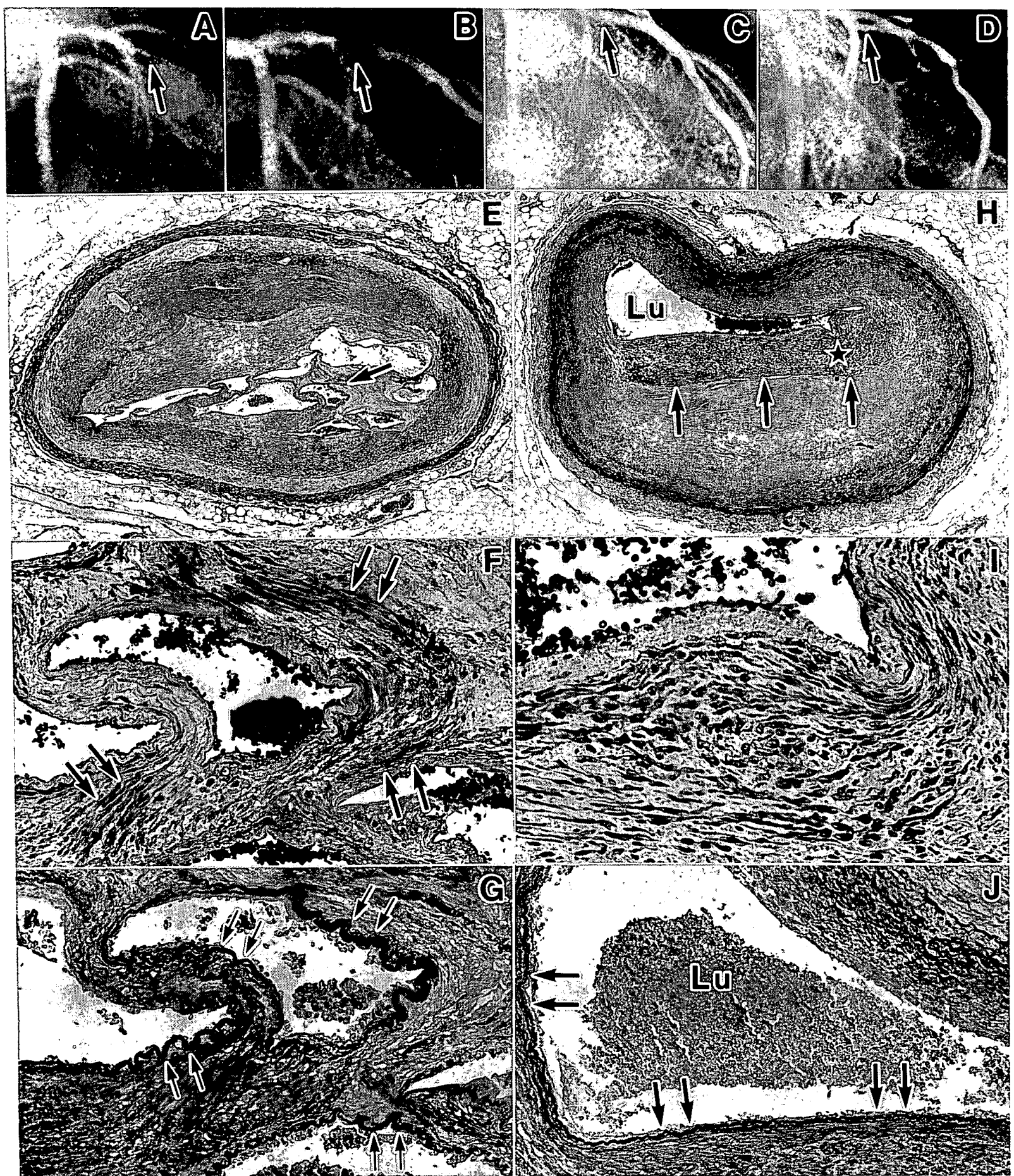

Fig.5. Case 13. This patient was admitted because of acute anterolateral myocardial infarction. A: Segment 7 of the left anterior descending artery (arrow) was completely occluded. B: After PTCR, PTCA was performed, achieving patency with stenosis of 50 to $75 \%$. C: Two weeks later, the stenosis was further reduced to 25 to $50 \%$. D: One year later a restenosis of 50 to $75 \%$ was found. E: Histological picture of the site of PTCA (Elastica van-Gieson stain). Many small lumina are seen. F: Magnified view of the portion in E indicated by the arrow (Masson's trichrome stain). Significant proliferation of smooth muscle cells (arrows) is observed. G: Magnified view of the portion in E indicated by the arrow (Elastica van-Gieson stain). Proliferation of relatively thick elastic fibers (arrows) is seen. H: Histological picture approximately $8 \mathrm{~mm}$ peripheral of E (Masson's trichrome stain). Significant proliferation of spindleshaped intimal cells is observed in the region adjacent to the lumen $(\mathrm{Lu})$, indicated by the arrows. I: Magnified view of the portion in $\mathrm{H}$ marked with a star. These cells were stained reddish-brown with Masson's trichrome, and reacted positively to immunohistochemical stain using anti-actin antibody. J: Magnified view of the portion in $\mathrm{H}$ adjacent to the lumen (Lu) (Elastica van-Gieson stain). Relatively thick elastic fibers are seen in the intima. 


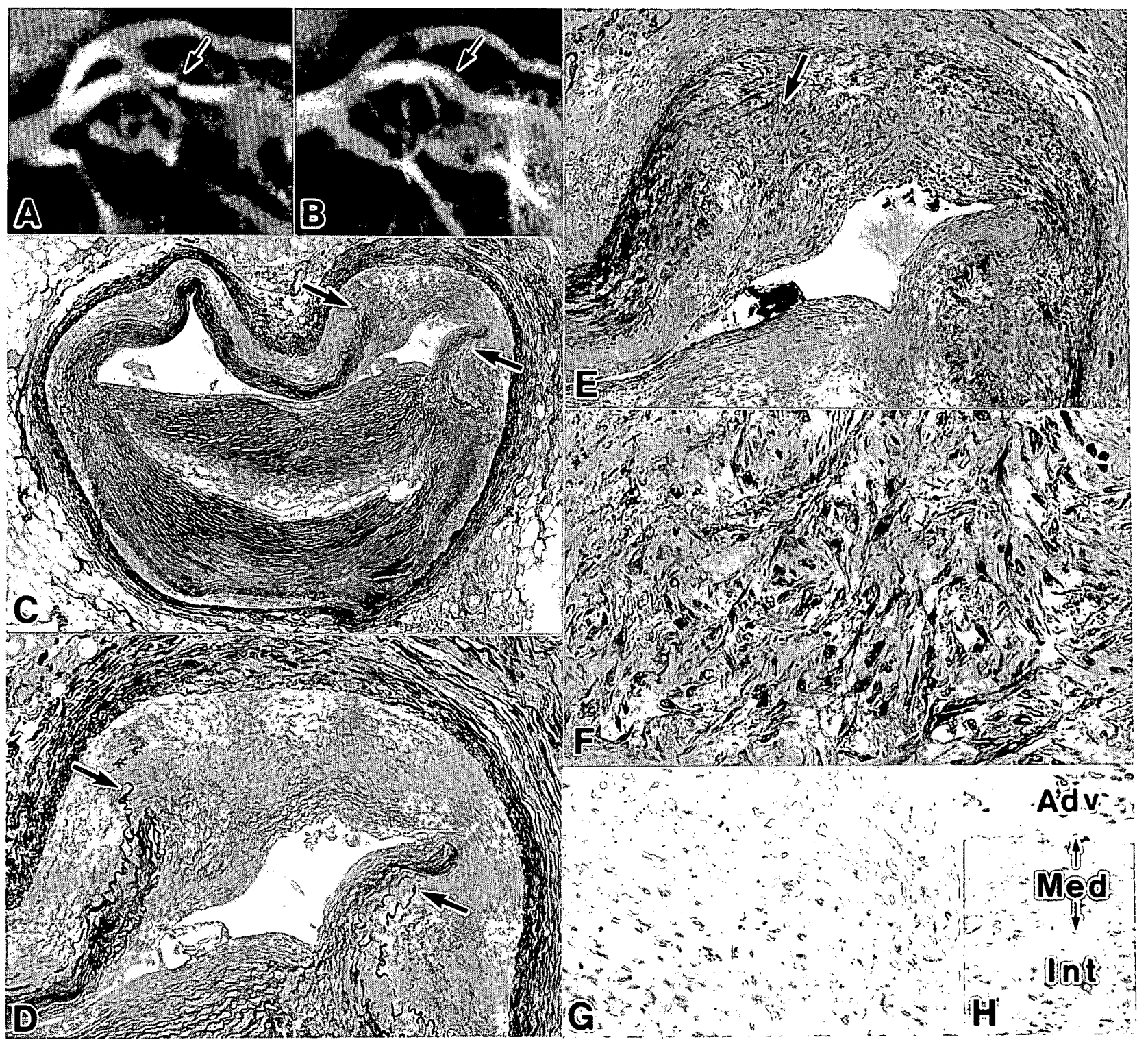

Fig.6. Case 17. A: $90 \%$ stenosis was seen in the left anterior descending artery. B: The stenosis was reduced to $10 \%$ by PTCA. The patient, however, died of pneumonia 4 months later. C: Histological picture of the site of PTCA (Elastica van-Gieson stain). Intimal and medial disruption is observed (arrows). $\mathrm{D}, \mathrm{E}$ : Magnified view of the portion in $\mathrm{C}$ indicated by the arrows (E: Masson's trichrome stain). A significant proliferation of intimal cells is seen. F: Magnified view $(\times 100)$ of the portion in $E$ indicated by the arrow. G: Intimal cells reacted positively with immunohistochemical stain using anti-actin antibodies. H: Coronary artery which did not undergo PTCA. The medial smooth muscle cells are shown to react positively with the anti-actin antibodies (Adv: adventitia, Med: media, Int: intima).

charged. She then developed dysphagia and 3 months later underwent surgery again for her aortic aneurysm. The patient died of pneumonia 1 month after this operation.

Autopsy findings: The PTCA site showed intimal and medial disruption in the wall opposite the portion with atheromatous plaque (arrows in Fig. 6C and D), as well as significant proliferation of intimal cells (Fig. 6E and F). These cells were identified as smooth muscle cells since they stained reddish-brown with Masson's trichrome and immunohistochemically positive with antiactin antibody (Fig. 6G). It was also found that the greater the fragmentation of internal elastic lamina, the more widespread was the proliferation of smooth muscle cells in the intima (Fig. 7).

Case 18: N.O. 73-year-old man

This patient with old anteroseptal myocardial infarction further developed unstable angina pectoris. Segment 7 of left anterior descending artery with 99\% stenosis (Fig. 8A) and segment 


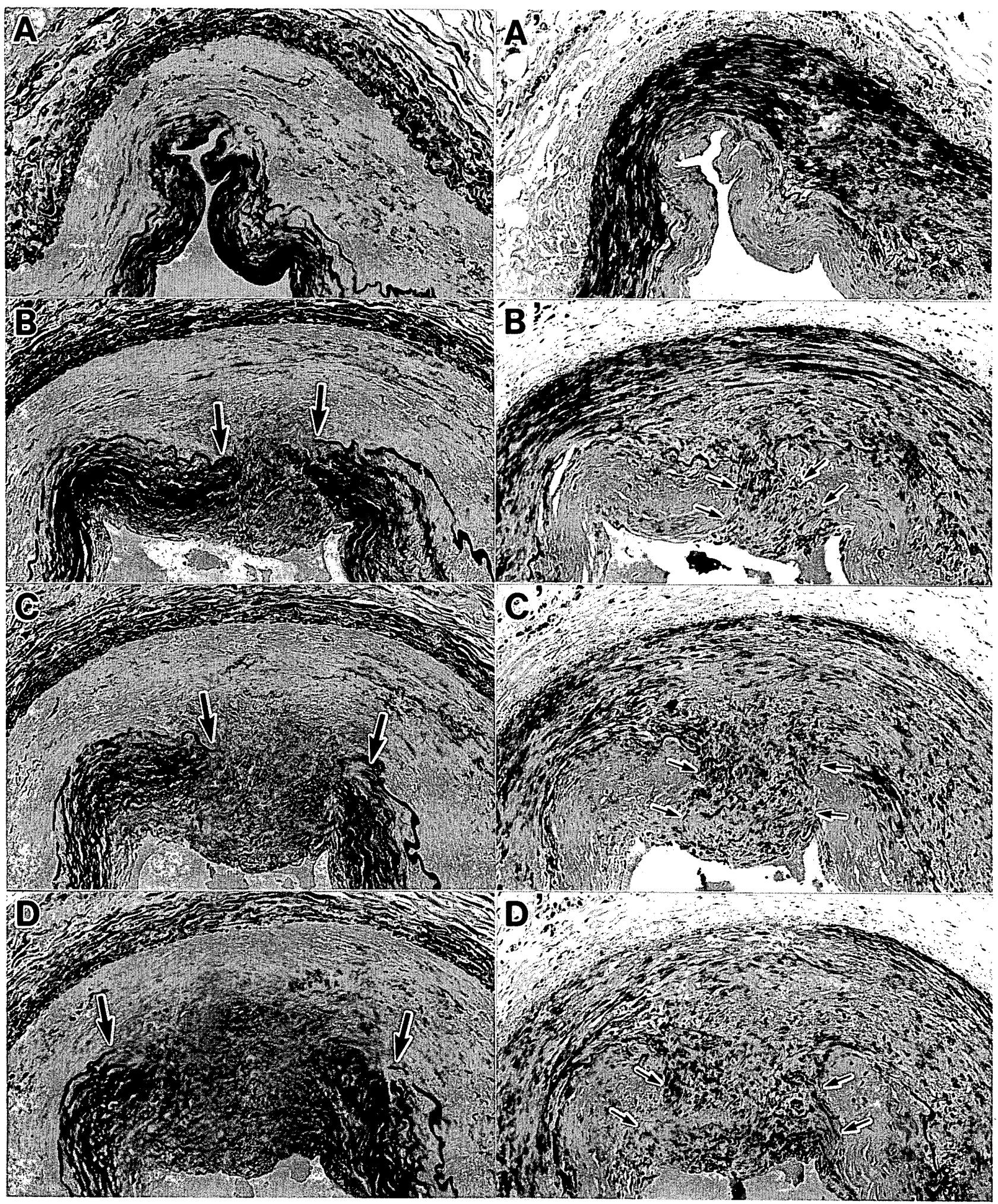

Fig.7. Case 17. Histological picture about $0.4 \mathrm{~mm}$ proximal to the portion shown in Fig. $6 \mathrm{C}$ (A to D: Elastica van-Gieson, A' to D': Masson's trichrome stain). A, B, C and D are sections cut at 40 to $80 \mu \mathrm{m}$ intervals. The relationship between fragmentation of internal elastic lamina and proliferation of smooth muscle cells is shown. In proliferation of smooth muscle cells, the internal elastic lamina serves as a barrier. The greater the fragmentation of the internal elastic lamina (arrows), the more widespread is the proliferation of smooth muscle cells in the intima (arrows).

11 of left circumflex artery with $75 \%$ stenosis were treated by PTCA (Fig. 8B, C). Although the stenoses were both reduced to about $25 \%$ by PTCA, 2 months later restenosis occurred in 

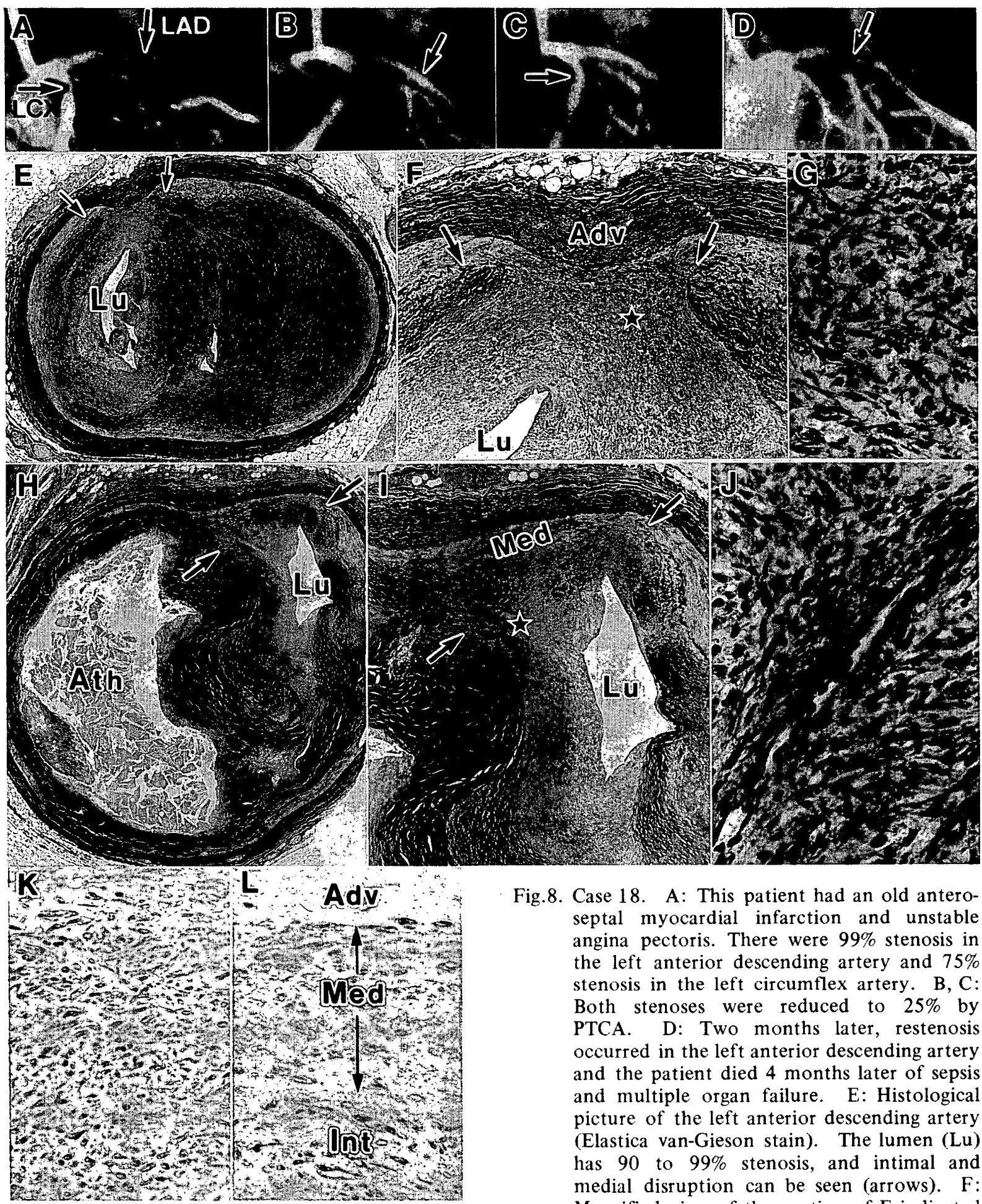

Fig.8. Case 18. A: This patient had an old anteroseptal myocardial infarction and unstable angina pectoris. There were $99 \%$ stenosis in the left anterior descending artery and $75 \%$ stenosis in the left circumflex artery. B, C: Both stenoses were reduced to $25 \%$ by PTCA. D: Two months later, restenosis occurred in the left anterior descending artery and the patient died 4 months later of sepsis and multiple organ failure. E: Histological picture of the left anterior descending artery (Elastica van-Gieson stain). The lumen (Lu) has 90 to $99 \%$ stenosis, and intimal and medial disruption can be seen (arrows). F: Magnified view of the portion of $E$ indicated by the arrows. It is seen that the coronary arterial wall was partly composed of only adventitia (Adv) immediately after PTCA. G: Magnified view of the part on F marked with a star (Masson's trichrome stain, $\times 100)$. Significant proliferation of spindle-shaped intimal cells, stained reddish-brown, is observed. H: Histological picture of the PTCA site in the left circumflex artery (Elastica van-Gieson stain). The lumen ( $\mathrm{Lu})$ has 90 to $99 \%$ stenosis. I: Magnified view of the $\mathrm{H}$ part indicated by arrows. Although the internal elastic lamina (arrows) is fragmented, there is still some residual media (Med). J: Magnified view of the portion in I marked with a star (Masson's trichrome stain, $\times 100$ ). The significant proliferation of intimal cells shows that restenosis has occurred by excessive proliferation of these cells. $\mathrm{K}$ : Immunohistochemical staining with anti-actin antibodies to identify spindle-shaped intimal cells gave a positive reacton. L: Coronary artery which did not undergo PTCA. The medial smooth muscle cells are shown to react positively to the anti-actin antibodies (Adv: adventitia, Med: Media, Int: intima). 
I. Early deaths ( 1 hour to 7 days)

1. Intimal desquamation, thrombus.

2. Intimal disruption and desquamation, thrombus.

3. Intimal disruption and desquamation, thrombus.

4. Medial dissection, intimal disruption, thrombus.

5. Intimal and medial disruption, fragmentation of IEL, medial dissection, intimal desquamation, thrombus.

6. Medial dissection, intimal disruption, thrombus.

7. Medial dissection, intimal disruption, thrombus.

8. Medial dissection, intimal disruption and desquamation, thrombus.

9. Medial dissection, intimal disruption and desquamation, thrombus.

II. Later deaths (16 days to 2 years and 11 months)

10. Intimal and medial disruption, fragmentation of IEL, medial dissection, proliferation of SMC.

11. Intimal desquamation, release of atheromatous plaque into lumen.

12. Proliferation of SMC and elastic fibers on the luminal side of intima, intimal disruption.

13. Proliferation of SMC and elastic fibers on the luminal side of intima.

14. Intimal and medial disruption, fragmentation of IEL, medial dissection, proliferation of SMC.

15. Intimal and medial disruption, fragmentation of $I E L$, proliferation of SMC.

16. Fibrous plaque.

17. Intimal and medial disruption, fragmentation of $I E L$, proliferation of $S M C$

18. (Left anterior descending artery) Intimal and medial disruption, fragmentation of IEL, proliferation of SMC. (left circumflex artery) Intimal and medial disruption, fragmentation of IEL, proliferation of SMC.

19. Intimal and medial disruption, fragmentation of IEL, proliferation of SMC.

20. Intimal and medial disruption, fragmentation of IEL, proliferation of SMC.

21. Intimal and medial disruption, fragmentation of IEL, proliferation of SMC.

$I E L=$ internal elastic lamina; $S M C=$ smooth muscle cells.

segment 7 (Fig. 8D). When a second PTCA was performed, dissection occurred in the left main truncus and an emergency aortocoronary bypass was performed. Two months later, however, the patient died of multiple organ failure as well as sepsis.

Autopsy findings: The lumen ( $\mathrm{Lu}$ ) of the left anterior descending artery where PTCA had been performed had 90 to $99 \%$ stenosis (Fig. 8E), and showed large intimal and medial disruptions (arrows in Fig. 8E and F), suggesting that the coronary arterial wall of this region immediately following PTCA consisted almost entirely of adventitia (Adv in Fig. 8F). The site of the restenosis (star in Fig. 8F) showed a significant proliferation of intimal cells, which stained reddish-brown with Masson's trichrome (Fig. 8G). In addition, the PTCA site of the left circumflex artery also showed 90 to $99 \%$ stenosis (Fig. $8 \mathrm{H}$ ), and fragmentation of the internal elastic lamina was observed (arrows in Fig. 8H and I). Unlike the aforementioned left anterior descending artery, there was some residual media (Med). As expected, a significant proliferation of intimal cells (Fig. 8J) was seen in the restenosed region (star in Fig. 8I). Immunohistochemical staining with anti-actin antibody for identification of spindle-shaped intimal cells was positive (Fig. 8K).

The above PTCA site findings have been summarized in Table II. All 9 patients who had died shortly after PTCA showed formation of fresh thrombus. Of the patients who had died considerably later, 8 (9 coronary arteries) showed fragmentation of internal elastic lamina. In these 9 arteries, a significant proliferation of intimal cells was observed mainly on the intimal side of the regions with fragmentation of internal elastic lamina. In 2 cases, proliferation of intimal cells and elastic fibers was observed on the 
A.

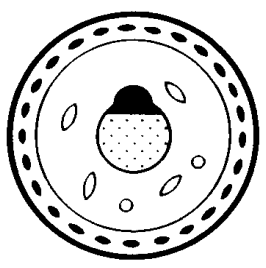

D.

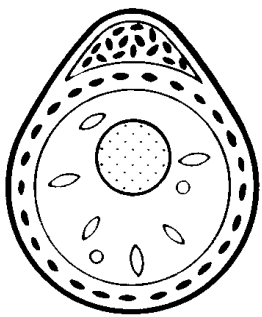

B.

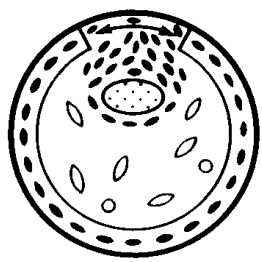

C.

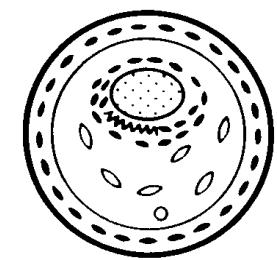

Lumen

A Thrombus

$\because$ Smooth Muscle Cells

mm Elastic Fiber

(S.Morimoto, 1987$)$

Fig.9. Morphology of restenosis after PTCA.
A: Thrombus formation.
B: Due to fragmentation of internal elastic lamina accompanying medial disruption, smooth muscle cells proliferate at the site.
C: No fragmentation of the internal elastic lamina is evident. Smooth muscle cells and elastic fibers proliferate in the intima around the lumen.
D: Smooth muscle cells proliferate in the false lumen.

luminal side of the intima, despite the absence of fragmentation in the internal elastic lamina. For identification, these intimal cells were immunohistochemically stained with anti-actin antibodies, producing a positive reaction in all 9 arteries. These spindle-shaped cells were identified as smooth muscle cells by their staining reddish-brown with Masson's trichrome and their positive immunohistochemical staining with antiactin antibody. In 2 cases with medial dissection (case 10, 14), proliferation of smooth muscle cells was found in false lumens.

\section{DISCUSSION}

Only 4 deaths were a direct result of complications associated with PTCA, and the findings obtained in this study were thought to show lesions common to PTCA in general. Although there have been several case reports ${ }^{7,9-15}$ on restenosis after PTCA, no report has until now appeared on histopathological study of restenosis based on as many as 21 autopsied hearts.

In this study the restenosis lesions were classified into 4 categories, as shown in the schematic drawings (Fig. 9).

A. Organization of thrombus: Fresh thrombus formation was found in all 9 early death cases in the present study (Table II). Block et $\mathrm{al}^{5}$ and Austin et $\mathrm{l}^{9}$ have also found thrombus formation in the intimal cracks of patients who died soon after PTCA. The possibility that such thrombus formation ${ }^{18}$ leads to irreversible stenosis of arteries cannot be ruled out. In other words, restenosis following PTCA may start to form very soon after PTCA.

B. Fragmentation of the internal elastic lamina and proliferation of smooth muscle cells: Examples similar to ours have been reported by Essed et al ${ }^{11}$ and Giraldo et al ${ }^{12}$ To understand the proliferation of these cells, it is helpful to keep in mind the "response to injury hypothesis of atherosclerosis" proposed by Ross et al, ${ }^{19,20}$ which is now generally recognized. According to the theory, when the endothelium of a vessel is injured and detached for some reason, first the platelets adhere to and aggregate at the damaged site; next, the platelets' $\alpha$ granules release platelet derived growth factor (PDGF), inducing medial smooth muscle cells to migrate to the intima where they proliferate, causing intimal hyperplasia. In PTCA, the balloon catheter injures the intima in a great majority of cases, and in some patients medial disruption occurs. This major fragmentation of the internal elastic lamina was considered to facilitate migration of smooth muscle cells and precipitate their proliferation. 
The mechanism of PTCA-induced luminal enlargement most frequently encountered is intimal and medial disruption in the wall located opposite the site with atheroma. ${ }^{4}$ Fragmentation of the internal elastic lamina occurs as a natural consequence of this. In the present investigation, proliferation of smooth muscle cells was observed on the intimal side around the site of fragmentation of the internal elastic lamina in 9 arteries from 8 patients with such fragmentation, who had died long after PTCA. As mentioned earlier, medial disruption is important in luminal enlargement in a way similar to the unhooping of a barrel. When this is accompanied by fragmentation of the internal elastic lamina, restenosis occurs, and this might be considered an intrinsic self-contradiction of PTCA.

C. Absence of fragmentation of the internal elastic lamina and presence of proliferation of smooth muscle cells in the intima around the lumen (case 12 and 13): Although it is difficult to determine whether proliferation of smooth muscle cells manifests simply a form of the original arteriosclerosis or a reaction to PTCA, it was considered reasonable to assume that some stimulus, i.e., PTCA, caused this because these cells were proliferating on the luminal side of the intima, and thick elastic fibers were seen at the site. Both cases, however, had myocardial infarction, and it is necessary to take into account the effect of thrombus organization. The morphology of $\mathrm{C}$ needs further study.

D. Medial dissection and proliferation of smooth muscle cells in the false lumen (case 10, 14): An example similar to ours has been reported by Essed et al, 1 who stated that a PTCA site showed a split of atheromatous plaque and medial dissection, and that a lumen with a false channel was almost totally occluded due to considerable proliferation of fibrocellular tissue. They inferred that medial dissection exposes smooth muscle cells directly to blood, promoting thrombus formation, and releasing PDGF, and that as a result of this, smooth muscle cells proliferate. When the aortic media is dissected, the false lumen may become occluded by proliferation of fibrocellular tissue undergoing restoration! It is interesting that a phenomenon found in the aorta was similarly seen in coronary arteries.

As stated above, although smooth muscle cells are essential for restoration of arterial walls, their excessive proliferation results in intimal hyperplasia, i.e., restenosis. Waller et al, on the other hand, have made a histopathological study on coronary arteries of 3 patients who died suddenly or accidentally 80,90 , and 150 days, respectively, after PTCA, despite luminal enlargement of coronary arteries obtained by the procedure. They staled that only common atherosclerotic plaques, including cholesterol clefts were found, and that no disruptions or dissections were observed in the walls of coronary arteries. We also encountered one patient (case 16), who showed only a fibrous plaque despite luminal enlargement being clinically observed. Two years and 8 months had elapsed since PTCA when we studied this patient. Whether such pathological changes can take place with the passage of time seems to be one problem that needs to be solved. As there are still many unknowns regarding the healing process after PTCA, i.e., restenosis and temporal correlation, further examinations of a large number of patients need to be made.

\section{Acknowledgements}

The authors thank Dr. R. Takanashi, Mitsui Memorial Hospital, Drs. T. Kasajima and T. Nishikawa, Tokyo Women's Medical College, Dr. M. Aoki, Tokyo Metropolitan Bokutoh Hospital, Dr. K. Okano, Osaka Police Hospital, Dr. M. Furumoto, Hyogo Brain and Heart Center at Himeji, Dr. M. Imai, Sakakibara Memorial Hospital and Dr. N. Imazeki, National Defence Medical College for kind access to autopsied hearts.

\section{REFERENCES}

1. GRÜNTZIG AR, SENNING A, SIEGNTHALER WE: Nonoperative dilatation of coronary-artery stenosis. Percutaneous transluminal coronary angioplasty. New Engl J Med 301 : 61, 1979

2. HOLMES DR, VLIETSTRA RE, SMITH HC, VETROVEC GW, KENT KM, COWLEY MJ, FAXON DP, GRUENTZIG AR, KELSEY SF, DETRE KM, VAN RADEN MJ, MOCK MB: Restenosis after percutaneous transluminal coronary angioplasty (PTCA): A report from the PTCA registry of the National Heart, Lung, and Blood Institute. Am J Cardiol 53: 77c, 1984

3. GUITERAS VAL P, BOURASSA MG, DAVID PR, BONAN R, CREPEAU J, DYRDA I, LESPERANCE J: Restenosis after successful percutaneous transluminal coronary angioplasty: The Montreal Heart Institute Experience. Am J Cardiol 60: $50 \mathrm{~B}, 1987$

4. MORIMOTO S, SEKIGUCHI M, ENDO M, HORIE T, KITAZUME H, KODAMA K, YAMAGUCHI T, OHNO M, KUROGANE H, FUJINO M, SHIMIZU $\mathrm{Y}$, MIZUNO K, CHINO M: Mechanism of luminal enlargement in PTCA and restenosis: A histopathological study of necropsied coronary arteries collected from various centers in Japan. Jpn Circ J 51: 1101,1987 
5. BLOCK PC, MYLER RK, STERTZER S: Morphology after transluminal angioplasty in human beings. $N$ Engl J Med 305: 282, 1981

6. SAFFITZ JE, ROSE TE, OAKS JB, ROBERTS WC: Coronary arterial rupture during coronary angioplasty. Am J Cardiol 51: 902, 1983

7. WALLER BF, GORFINKEL HJ, ROGERS FZ, KENT KM, ROBERTS WC: Early and late morphologic changes in major epicardial coronary arteries after percutaneous transluminal coronary angioplasty. Am J Cardiol 53: 42C, 1984

8. MIZUNO K, KURITA A, IMAZEKI N: Pathological findings after percutaneous transluminal coronary angioplasty. Br Heart J 52: 588, 1984

9. AUSTIN GE, RATLIFF NB, HOLLMAN J, TABEI S, PHILLIPS DF: Intimal proliferation of smooth muscle cells as an explanation for recurrent coronary artery stenosis after percutaneous transluminal coronary angioplasty. J Am Coll Cardiol 6: 369, 1985

10. SOWARD AL, ESSED CE, SERRUYS PW: Coronary arterial findings after successful percutaneous transluminal coronary angioplasty. Am J Cardiol 56: 794,1985

11. ESSED CE, BRAND MVD, BECKER AE: Transluminal coronary angioplasty and early restenosis. Br Heart J 49: 393, 1983

12. GIRALDO AA, ESPOSO OM, MEIS JH: Intimal hyperplasia as a cause of restenosis after percutaneous transluminal coronary angioplasty. Arch Pathol Lab Med 109: 173, 1985

13. MORAIS CF, LOPES EA, CHECCHI H, ARIE S, PILEGGI F: Percutaneous transluminal coronary angioplasty - Histopathological analysis of nine necropsy cases. Virchows Arch A 410: 195, 1986

14. TOJO O, ARAI H, SAITO S, NAKAJIMA H, KUBORI S, KITAOKA T, SHIMIZU Y, MORIMURA Y, OGAWA N, UEDA M, FUJIMOTO T: Clinical and pathologic features of a patient who died of acute myocardial infarction 3 months after successful PTCA. Heart 19: 458, 1987 (in Japanese).

15. KOHCHI K, TAKEBAYASHI $S$, BLOCK PC, HIROKI T, NOBUYOSHI M: Arterial changes after percutaneous transluminal coronary angioplasty: Results at autopsy. J Am Coll Cardiol 10: 592,1987

16. HSU SM, RAINE L: The use of avidin-biotinperoxidase complex (ABC) in diagnostic and research pathology. In Advances in Immunohistochemistry, ed by DeLELLIS RA, Masson Publishing, New York, 1984, p31

17. WARNKE R, LEVY $R$ : Detection of $T$ and $B$ cell antigens with hybridoma monoclonal antibodies: A biotin-avidin-horseradish peroxidase method, $J$ Histochem Cytochem 28: 771, 1980

18. CHESEBRO JH, LAM JYT, BADIMON L, FUSTER V: Restenosis after arterial angioplasty: A hemorrheologic response to injury. $A m J$ Cardiol 60: 10B, 1987

19. ROSS R, GLOMSET JA: The pathogenesis of atherosclerosis (first of two parts). New Engl J Med 295: 369, 1976

20. ROSS R, GLOMSET JA: The pathogenesis of atherosclerosis (second of two parts). New Engl J Med 295: 420, 1976 